\title{
Preparing to Reach: Selecting an Adaptive Long-Latency Feedback Controller
}

\author{
Mohammad Ali Ahmadi-Pajouh, ${ }^{1,2}$ Farzad Towhidkhah, ${ }^{2}$ and Reza Shadmehr ${ }^{1}$ \\ ${ }^{1}$ Laboratory for Computational Motor Control, Department of Biomedical Engineering, The Johns Hopkins School of Medicine, Baltimore, Maryland 21205, \\ and ${ }^{2}$ Biomedical Engineering Department, Amirkabir University of Technology, Tehran, Iran 15875-4413
}

In a voluntary movement, the nervous system specifies not only the motor commands but also the gains associated with reaction to sensory feedback. For example, suppose that, during reaching, a perturbation tends to push the hand to the left. With practice, the brain not only learns to produce commands that predictively compensate for the perturbation but also increases the long-latency reflex gain associated with leftward displacements of the arm. That is, the brain learns a feedback controller. Here, we wondered whether, during the preparatory period before the reach, the brain engaged this feedback controller in anticipation of the upcoming movement. If so, its signature might be present in how the motor system responds to perturbations in the preparatory period. Humans trained on a reach task in which they adapted to a force field. During the preparatory period before the reach, we measured how the arm responded to a pulse to the hand that was either in the direction of the upcoming field, or in the opposite direction. Reach adaptation produced an increase in the long-latency (45-100 ms delay) feedback gains with respect to baseline, but only for perturbations that were in the same direction as the force field that subjects expected to encounter during the reach. Therefore, as the brain prepares for a reach, it loads a feedback controller specific to the upcoming reach. With adaptation, this feedback controller undergoes a change, increasing the gains for the expected sensory feedback.

\section{Introduction}

A model of motor control posits that, during voluntary movements, the brain engages a feedback controller that transforms sensory states into motor commands (Todorov and Jordan, 2002; Shadmehr and Krakauer, 2008). A crucial prediction of this model is that the feedback gains, defined as the map from sensory states into motor commands, should depend on dynamics of the task. For example, Diedrichsen (2007) measured the feedback response of the arm during reaching. In one condition, there were two cursors and each arm controlled its own cursor, while in another condition there was a single cursor that was controlled by both arms. When the right arm was perturbed, in the former condition the response was limited to the right arm, whereas in the latter condition both arms responded. In another example, Burdet et al. (2001) and others (Franklin et al., 2007; Wong et al., 2009) imposed a diverging force field on reaching movements and found that, with practice, perturbation response of the arm during the reach increased along an axis that was parallel to the diverging field. Finally, when people reached in a curl field in which the forces pushed the hand in only one direction, response

Received Aug. 19, 2011; revised May 17, 2012; accepted May 22, 2012.

Author contributions: M.A.A.-P. and R.S. designed research; M.A.A.-P. performed research; F.T. contributed unpublished reagents/analytic tools; M.A.A.-P. analyzed data; M.A.A.-P. and R.S. wrote the paper.

This work was supported by National Institutes of Health Grant NS057814, and a grant from the Human Frontiers

Science Foundation. We thank Adrian Haith for fruitful discussions and help with coding the experiment, and Michelle Harran for help with data collection.

Correspondence should be addressed to Reza Shadmehr, The Johns Hopkins School of Medicine, 410 Traylor Building, 720 Rutland Avenue, Baltimore, MD 21205. E-mail: shadmehr@jhu.edu.

DOI:10.1523/JNEUROSCI.4275-11.2012

Copyright $\odot 2012$ the authors $\quad 0270-6474 / 12 / 329537-09 \$ 15.00 / 0$ of the arm during the reach increased for perturbations that were parallel to the field (Wang et al., 2001; Kimura et al., 2006; Kimura and Gomi, 2009). This increased gain was present only in the long-latency period, $\sim 80 \mathrm{~ms}$ after the perturbation onset (Wang et al., 2001; Kimura and Gomi, 2009). Disruption of the motor cortex eliminated this change (Kimura et al., 2006). Together, these results suggest that, during reaching, the brain engages a feedback controller with gains that depend on dynamics of the task. The ability to adapt this feedback controller as a consequence of change in task dynamics depends on the integrity of the motor cortex.

Here, we wondered whether a signature of this feedback controller might be present in the preparatory period before the reach. A fundamental question is with regard to the computations that the brain performs during the preparatory period. Previous work shows that if a movement awaits a go cue, during the preparatory period the brain has largely programmed the motor commands, as these commands can be released via startling stimuli (Carlsen et al., 2008; Carlsen and Mackinnon, 2010). However, control of a movement involves formation of not just the motor commands, but a feedback control policy. Does the brain load the feedback controller during the preparatory period? We approached this hypothesis by training people on an instructed delay task in which they waited to reach to a target. We occasionally perturbed their hand during the preparatory period and measured the feedback response. After they had adapted their reaching movements in a curl field, we again measured the feedback gains during the preparatory period. We found a signature of the adapted reach controller in the preparatory period. 


\section{Materials and Methods}

A total of 67 healthy individuals participated in our study ( $18-45$ years of age; 34 females). Experimental procedures were approved by The Johns Hopkins University School of Medicine Institutional Review Board, and all subjects signed a consent form.

Experiment 1 . Subjects $(n=48)$ performed a reaching task while holding the handle of a planar robotic manipulandum and adapted to a standard curl force field. Their upper arm was supported by an air-sled. The subjects were divided into three groups. One group adapted to a clockwise velocity-dependent curl field (CW) $(n=15 ; \boldsymbol{f}=\boldsymbol{B} \dot{\boldsymbol{x}}$, where $B=[0,15 ;-15,0] \mathrm{N} \cdot \mathrm{s} / \mathrm{m}$ and $\dot{\boldsymbol{x}}$ is hand velocity vector). Another group adapted to a counterclockwise field (CCW) $(n=18 ; B=[0,-15 ; 15,0]$ $\mathrm{N} \cdot \mathrm{s} / \mathrm{m})$. A third group $(n=15)$ served as control and reached only in null field. Our purpose was to quantify the changes that accompanied this adaptation in terms of response to sensory feedback during the preparatory period before onset of the reach.

Subjects had no direct visual feedback of their hand or the robotic arm. Instead, a video projector painted a horizontal white screen directly above the plane of the hand. The arm was positioned at a configuration of $\left(30,90^{\circ}\right)$ representing the shoulder and relative elbow angles. The target appeared at $135^{\circ}$ at a distance of $14 \mathrm{~cm}$ (see Fig. $1 \mathrm{~A}$ ). Subjects heard three beeps, $100 \mathrm{~ms}$ in duration, separated by $500 \mathrm{~ms}$. They were instructed to start their reach with the third beep (see Fig. $1 B$ ). To enforce this, an invisible circle of $5 \mathrm{~mm}$ radius was placed on the start point, and they had to cross this boundary within the $100 \mathrm{~ms}$ duration of the third beep. The reach was rewarded with an "explosion" if it started within the window of the third beep and arrived at the target between 300 and $500 \mathrm{~ms}$. The experimental session was divided into nine blocks (see Fig. 1C): baseline, adaptation, and washout (each three blocks). Subjects were given 30 s rest periods between the blocks. Blocks 1-3 were in a null field, blocks 4-6 were in a curl field (except for a control group which only trained in null field), and blocks 7-9 were again in a null field. To compensate for the increased forces that subjects in the CW and CCW groups produced compared with the control group (which may result in fatigue), we increased the number of trials for the control group by $15 \%$ (compared with the other two groups) in blocks $4,5,7$, and 8 (all blocks for the control group were in the null field).

On random trials during the third, sixth, and ninth blocks, we assayed the feedback controller via a $50 \mathrm{~ms}, 7 \mathrm{~N} \mathrm{CW}$ or CCW force pulse directed toward 45 or $225^{\circ}$, respectively. The CW pulse was aligned with the direction of the CW field, and the CCW pulse was aligned with the direction of the CCW field. These pulses were given on randomly selected trials (probability of 0.27 ) during the preparatory period before the reach onset, either at 750 or $350 \mathrm{~ms}$ before the third beep. The force field was present only during the reach and not in the preparatory period. That is, the pulses were given under identical conditions in the baseline, adapt, and washout periods. The response was a displacement of the hand. We computed the component of the displacement that was parallel to the direction of the force pulse, and report that quantity here.

We recorded hand position and velocity at $200 \mathrm{~Hz}$. We recorded EMG from four muscles (short and long heads of biceps, lateral and long heads of triceps) at $1000 \mathrm{~Hz}$ (Experiment 1) or $2000 \mathrm{~Hz}$ (Experiment 2) using active electrodes that housed preamplifiers on the recording heads (Delsys). EMG data reported here are for short head of biceps and lateral head of triceps, as the other two muscles behaved very similarly. Reach onset was computed using a velocity threshold of $33 \mathrm{~mm} / \mathrm{s}$.

To compare EMG signals between subjects, after completion of the main task, subjects performed an additional task in which they produced a control force. In this task, a trapezoidal-shaped $9 \mathrm{~N}$ force $(5 \mathrm{~s}$ in duration, including $1 \mathrm{~s}$ rise and fall time) was applied to the hand at $225^{\circ}$ (engaging triceps) or $45^{\circ}$ (engaging biceps). The root-mean-squared (RMS) values of the resulting triceps and biceps EMG signals over the peak force period were used to normalize the EMG signals that we had recorded during the main task.

The DC component of the EMG signals was removed, the signal was low-pass filtered (four-pole Butterworth filter with $250 \mathrm{~Hz}$ cutoff frequency) and then rectified. The envelope of the rectified signal was detected using a moving window with bin size of $3 \mathrm{~ms}$. To find the short- and long-latency component of the EMG response to a pulse, M1 (20-45 $\mathrm{ms}), \mathrm{M} 2$ (45-75 ms), M3 (75-100 ms), and voluntary (100-180 ms) intervals were selected based on pulse onset.

Statistical analysis. To determine whether adaptation altered the feedback response, we performed a three-way repeated-measure ANOVA. For example, consider a data set composed of a baseline condition and an adapted condition. In each condition, we have a pulse at $-750 \mathrm{~ms}$ and at $-350 \mathrm{~ms}$. The pulses come in two directions: a CW pulse, and a CCW pulse. Therefore, we have three main effects: pulse time $(-750$ or -350 $\mathrm{ms}$ ), pulse type (CW or CCW), and condition (baseline, adapted). If there is a pulse type by condition interaction, then we conclude that the effect of the pulse type differed by condition. We followed this up with a post hoc test (Tukey's) to see whether the pulse that was in the direction of the force field produced a smaller response in the adapted condition versus the baseline condition.

Experiment 2. Our idea in this second experiment was to ask whether subjects could learn to alter their feedback response in the delay period based on contextual cues (target direction). Subjects $(n=19)$ were trained to reach to targets that were placed at $10 \mathrm{~cm}$ with respect to a start location (see Fig. 5A). They encountered a velocity-dependent curl field (strength of $20 \mathrm{~N} \cdot \mathrm{s} / \mathrm{m}$ ). The field pushed the hand to the left for target 1 , and to the right for target 2 . The target sequence was random. As in Experiment 1, they heard three consecutive beeps (separated by $500 \mathrm{~ms}$ ) and were trained to start their reach on the third beep. On randomly selected trials, they received a force pulse to the hand at $450 \mathrm{~ms}$ before the third beep. This pulse was directed at either $45^{\circ}$ (R-pulse) or $225^{\circ}$ (Lpulse), selected at random. The experiment had nine blocks. Blocks 1-3 were in the null field, and blocks 3-9 were in the force field (see Fig. 5B). First and second blocks were 120 and 60 trials, respectively. All other blocks contained 150 trials. After completion of this task, subjects performed an additional task in which they produced a control force. In this task, a trapezoidal-shaped $15 \mathrm{~N}$ force $(5 \mathrm{~s}$ in duration, including $1 \mathrm{~s}$ rise and fall time) was applied to the hand at $225^{\circ}$ (engaging triceps) or $45^{\circ}$ (engaging biceps). The RMS values of the resulting triceps and biceps EMG signals over the peak force period were used to normalize the EMG signals that we had recorded during the main task. All other aspects of the task were similar to Experiment 1. We removed trials in which the reach started before the go cue, or the hand was not stationary during the time of the perturbation ( $\sim 7 \%$ of the trials).

\section{Results}

We hypothesized that, in anticipation of making a reaching movement, the brain prepares a feedback controller that takes into account the dynamics of the upcoming movement. To test our hypothesis, in Experiment 1, we probed the feedback response via a force pulse to the hand during the preparatory period before movement onset in three conditions: a baseline condition in which the reaching movements were in a null field, an adapted condition in which the reaching movements were in a force field, and a washout condition in which the dynamics had returned to null.

We probed the feedback response at two time points during the preparatory period $(-750$ and $-350 \mathrm{~ms}$; Fig. $1 \mathrm{~B})$, during three conditions (baseline, adapt, and washout; Fig. 1C), and with two types of pulses (CW and CCW). The feedback response (hand displacement) for a representative subject is shown in Figure $1 \mathrm{D}$. The pulse produced a smaller displacement of the hand at $-350 \mathrm{~ms}$ versus $-750 \mathrm{~ms}$, suggesting that the gain of the feedback system was larger near movement onset. Importantly, this gain changed with adaptation. After the subject had trained in a CCW force field, a CCW pulse produced a smaller displacement of the hand with respect to the baseline period. After washout, the feedback response had returned to near-baseline levels. In comparison, a CW pulse produced a displacement that was comparable in baseline, adapt, and washout periods. That is, adaptation to a force field coincided with an asymmetric change in the gain 
A
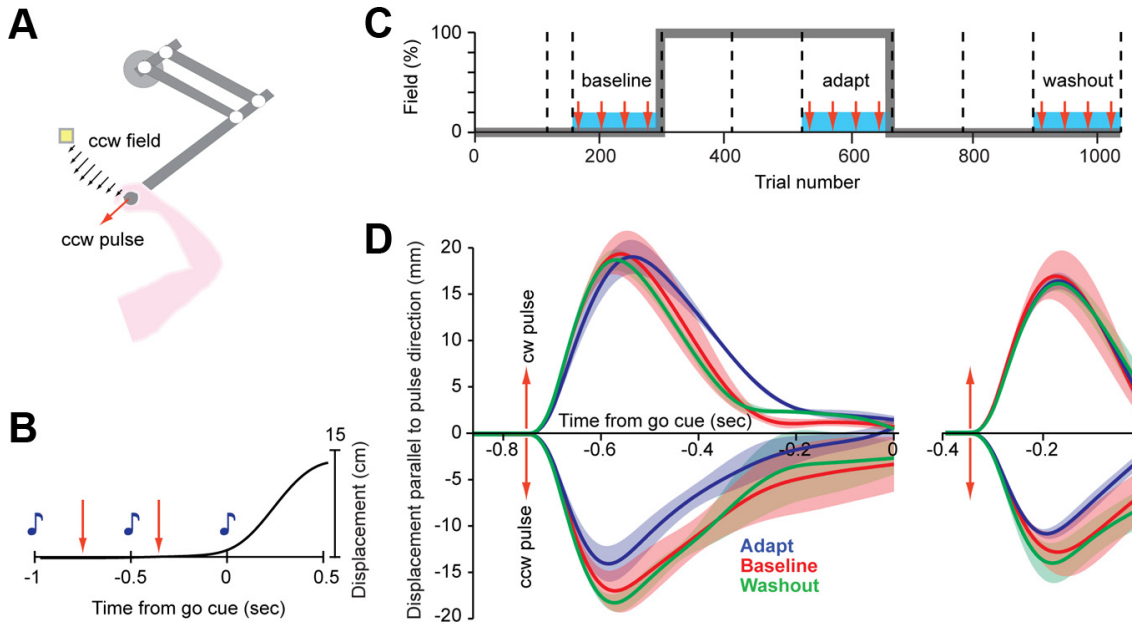

Figure 1. Protocol for Experiment 1 and results from a representative subject. $A$, The subject held the handle of a manipulandum and reached to a target. A velocity-dependent curl force field was applied to the hand during the reach period only. On occasional trials, a force pulse was applied to the hand during the preparatory period before the reach to quantify the state of the feedback controller of the arm. We asked whether this controller changed as the subject adapted their reaching movements in the field. $\boldsymbol{B}$, On each trial, the subject heard three beeps ( $100 \mathrm{~ms}$ in duration) and was instructed to start the reach in such a way so that during the third beep the hand crossed the boundary of an imaginary $5 \mathrm{~mm}$ radius circle centered on the start position. The arrows indicate onset time of the $50 \mathrm{~ms}$ duration force pulses that were occasionally given during the preparatory period. C, The gray line shows the state of the force field during the reaching movements. The dashed lines represent set breaks. The arrows represent the three periods in which we measured the feedback response by giving force pulses on occasional trials. $\boldsymbol{D}$, Feedback response of a representative subject who trained in a CCW field. This plot shows hand displacement parallel to the direction of the pulse. The upward and downward displacements represent responses to $\mathrm{CW}$ and $\mathrm{C} C \mathrm{~W}$ pulses. The left figure shows the average response to a pulse at $-750 \mathrm{~ms}$. The right figure shows the average response to a pulse at $-350 \mathrm{~ms}$. The arrow indicates onset of the pulse. This subject showed an increased gain in the adapted state for CCW pulses but not $\mathrm{CW}$ pulses. Error bars are SEM.

of the feedback response in the preparatory period: the gain increased for perturbations that displaced the hand in the direction of the field, but did not change for perturbations in the opposite direction.

\section{Reach-related changes}

Figure 2 is a summary of reach data during various conditions. Movements were to a single target. As a consequence, imposition of the field produced errors that within $\sim 30$ trials were reduced to near-baseline levels (maximum perpendicular displacement; Fig. $2 \mathrm{~A}$ ). Because timing of the go-cue was predictable (the third beep), subjects started their movements slightly before the cue. Using a velocity threshold, we estimated that, on average, the reach started at $137.2 \pm 10 \mathrm{~ms}$ before the go cue (mean \pm SEM). Importantly, reach start times were stable across the various conditions (Fig. $2 B, C$ ). Figure $2 C$ shows hand displacement in the direction of the target, and Figure $2 D$ shows muscle activation patterns. With repetition of the reaching movements in the field, the brain learned to increase the activity of muscles that could counter the field. Training in the CW field produced increased activation of biceps near movement onset, and exposure to the CCW field produced increased activation of triceps near movement onset. In comparing the EMG signals in the adapted and baseline conditions, the earliest changes in biceps and triceps EMG were at around $-200 \mathrm{~ms}$ (Fig. 2D). This is crucial because it implies that, at the time of the pulse ( -750 or $-350 \mathrm{~ms})$, these muscles were at comparable states in the baseline, adapt, and washout conditions.

\section{Preparatory period changes in feedback response}

Figure 3 is a summary of how the arm responded to force pulses in the preparatory period. Using a three-way repeated-measure
ANOVA, we compared the peak displacement produced by the pulses as a function of condition (baseline and adapt), pulse type (CW and CCW), and time $(-750$ and $-350 \mathrm{~ms})$. For the subjects who trained in the CW field (Fig. $3 A$ ), the pulse produce a smaller displacement at -350 $\mathrm{ms}$ versus $-750 \mathrm{~ms}$ (main effect of pulse time, $\left.F_{(1,14)}=48.9 ; p<0.0001\right)$. We found a main effect of condition $\left(F_{(1,14)}=\right.$ $7.9 ; p<0.015)$, and a condition by pulse type interaction $\left(F_{(1,14)}=4.63 ; p<0.05\right)$. Existence of a condition by pulse interaction suggests that the effect of pulse type differed by condition. Indeed, a post hoc analysis showed that, for subjects who reached in the $\mathrm{CW}$ field, the $\mathrm{CW}$ pulse produced a smaller displacement of the hand in the adapt condition compared with the baseline condition $(p<0.01)$. In contrast, the CCW pulse produced comparable displacements in the adapt and baseline conditions $(p>0.13)$. We made similar observations for the subjects who trained in the CCW field (Fig. $3 B)$. For the CCW group, the displacements were smaller at -350 ms versus $-750 \mathrm{~ms}$ (main effect of pulse time, $\left.F_{(1,17)}=46, p<0.0001\right)$. We again found a condition by pulse type interaction $\left(F_{(1,17)}=5.86 ; p<0.03\right)$. A post hoc analysis showed that, for subjects who reached in the CCW field, the CCW pulse produced a smaller displacement of the hand in the adapt condition compared with the baseline condition $(p<$ $0.05)$. In contrast, the CW pulse produced comparable displacements in the two conditions $(p>0.2)$.

Experiment 1 included a control group of subjects who trained only in the null field (Fig. $3 C$ ). We observed that the -350 $\mathrm{ms}$ pulse produced a smaller displacement than the $-750 \mathrm{~ms}$ pulse (main effect of pulse time, $F_{(1,14)}=8.01 ; p<0.006$ ). However, unlike the CW and CCW groups, the displacement did not differ in the various conditions. There was no main effect of condition $\left(F_{(1,14)}=0.16 ; p>0.69\right)$, no effect of probe type $\left(F_{(1,14)}\right.$ $=0.22 ; p>0.64)$, and no interaction between probe and condition $(p>0.83)$. In summary, during the preparatory period, the gain of the sensory-motor feedback loop was higher for the pulse near movement onset. With training in a force field, this feedback gain increased for the pulse that pushed the hand in the same direction as the field.

An important question is regarding the neural basis of the change in the feedback gain. A sensitive measure of the feedback response is EMG, as it allows for a precise estimate of change in the neural response. The timing of the change in EMG from one condition to another is a particularly useful proxy for inferring whether the neural changes had a basis in the spinal short-latency circuits, or supraspinal long-latency circuitry. Figure 4 summarizes the EMG that we recorded in response to the force pulses. In Figure $4 A$, we have plotted the across-subject averaged response in the adapt and baseline conditions. The CW pulse engaged the biceps and the CCW pulse engaged the triceps. When the pulse was in the same direction as the field, activity in biceps in the CW group was larger in adapt versus baseline conditions, and activity in triceps in the CCW group was larger in adapt versus baseline 

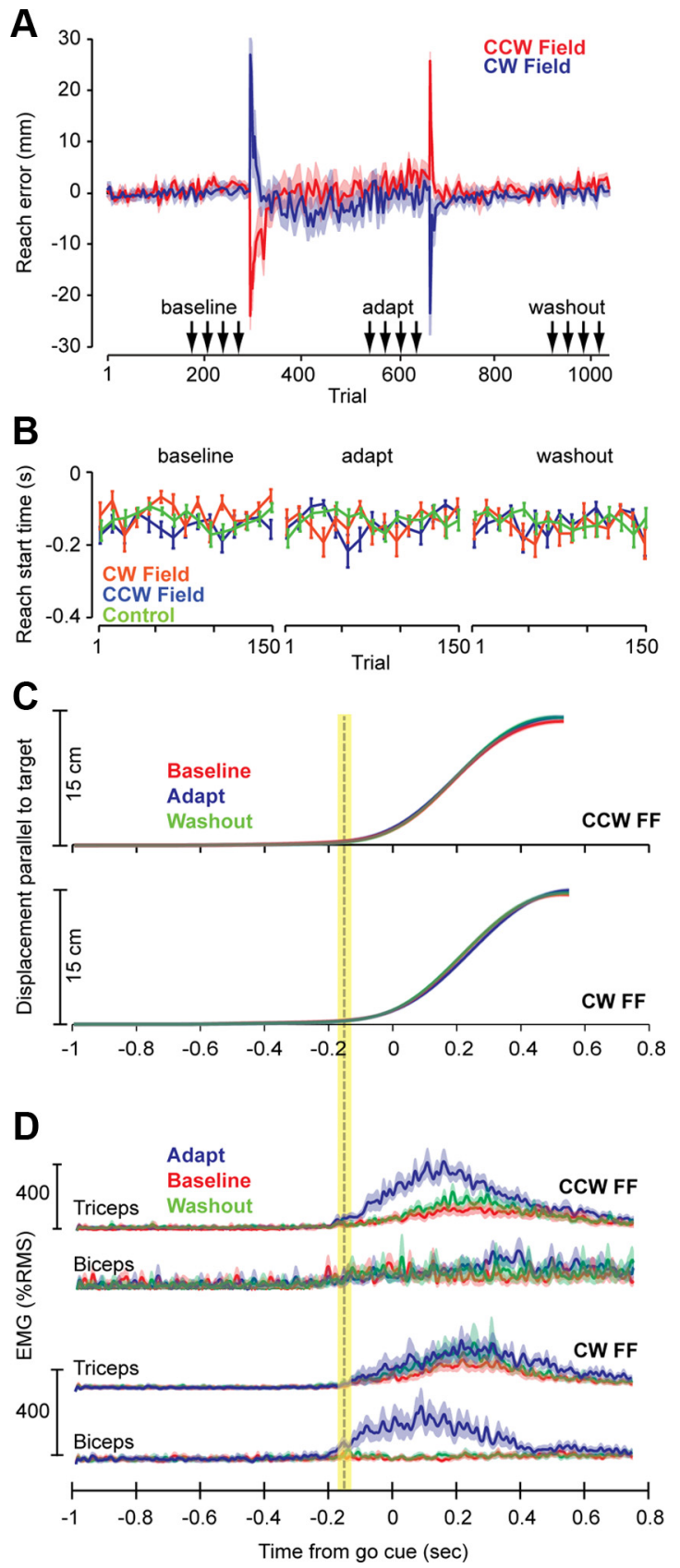

Figure 2. Performance during reaching movements. $\boldsymbol{A}$, The plot shows a measure of error during reaching (maximum displacement perpendicular to the target direction) for subjects in the $\mathrm{CW}$ and $\mathrm{CCW}$ groups (error bars are SEM). The arrows mark the range of trials in which we probed the feedback gains in the preparatory period via force pulses. Bin size is 5 , except for the first 10 trials of the adapt and washout blocks, in which bin size is 2 (to highlight the rapid changes). $\boldsymbol{B}$, Reach start times with respect to the go cue (third beep) are plotted for the CW, $\mathrm{CCW}$, and control groups. The baseline, adapt, and washout periods refer to the trials marked with arrows in $\boldsymbol{A}$. Bin size is 10. Error bars are SEM. C, Displacement of the hand parallel to the target direction is plotted for the CW and CCW groups in the baseline, adapt, and washout periods. Error bars are SEM (they are very small). The dashed vertical line marks across-subject averaged reach start time, and the yellow bar indicates SEM. D, Muscle activations during reaching were normalized for each subject with respect to the activity of that muscle in a separate task in which a known force was gradually imposed on the hand (see Materials and Methods). The term RMS refers to the root-mean-squared value of EMG from that muscle during this control task. This plot shows across-subject averaged EMG activity from two muscles in the reach trials. After subjects adapted to the CCW force field, triceps EMG showed a marked increase near reach onset. After subjects adapted to the CW force field, biceps EMG showed a marked increase near reach onset. Error bars are SEM. conditions. In contrast, when the pulse was in the opposite direction of the field, the EMG response from biceps and triceps appeared unchanged in the adapt versus baseline conditions. To quantify the timing and magnitude of these changes, we did a within-subject comparison of the EMG response in the adapt and baseline conditions. For each subject, we subtracted the EMG response in the pulsed trials in the baseline condition from the pulsed trials in the adapt condition (Fig. $4 B$ ). We then divided the results into four time bins with respect to the onset of the force pulse: $20-45 \mathrm{~ms}$ (M1), 45-75 ms (M2), 75-100 ms (M3), and 100-180 ms (voluntary) (Fig. 4C). For the subjects in the CW group, we found robust increases in the response of the biceps muscle at $75-100 \mathrm{~ms}$ for both the -750 and $-350 \mathrm{~ms}$ CW pulses (two-way ANOVA with pulse time and response bin as main effects, main effect of response bin with $p<0.0001$, no other main effects and no interactions). For the subjects in the CCW group, we found robust increases in the triceps muscle at 75-100 $\mathrm{ms}$ for both the -750 and $-350 \mathrm{~ms}$ CCW pulses (two-way ANOVA with pulse time and response bin as main effects, main effect of response bin with $p<0.014$, no interactions). Therefore, reach adaptation did not alter the short-latency response to a force pulse during the preparatory period. Rather, it affected the gain of the long-latency response, increasing it for displacements that were in the same direction as the field. Finally, our comparison of washout and baseline conditions found no significant differences in any muscle, suggesting that by the end of washout the adaptation-driven changes in feedback response had returned to baseline.

\section{Did the pulse release the motor commands that start the reach?}

If a startling stimulus is given during the delay period in which the brain awaits a go cue, the brain often releases the motor commands that initiate the movement (Carlsen et al., 2008; Carlsen and Mackinnon, 2010). Therefore, perhaps the pulse that we gave during the delay period acted as a startling stimulus, releasing a sequence of motor commands. Is there evidence that our results reflect a change in feedback response and not merely a premature release of the movement? First, as the data in Figures 3 and 4 illustrate, only the response to a pulse that was in the same direction as the upcoming field showed a change with adaptation. That is, only when the arm was stretched during the delay period in the same direction as it was expected to be stretched during the reach did we observe a change in the response. The fact that the response showed adaptation only for one of the two pulses appears inconsistent with the hypothesis that the pulses resulted in a premature release of the motor commands. Second, the data in Figure $4, A$ and $B$, demonstrate that the EMG response to a pulse showed a transient increase, and then a decline back to prepulse levels. If the pulse had triggered the motor commands that initiate the movement, the EMG would resemble that shown in Figure $2 B$, where we see that the traces increase before movement onset and remain large for many hundreds of milliseconds. In summary, the data in Figure 3, $A$ and $B$, suggest that the pulse during the preparatory period (at least at $-750 \mathrm{~ms}$ ) is a reasonable proxy for the feedback controller, as it does not trigger initiation of the movement toward the target.

\section{Contextual selection of the feedback policy}

The results of Experiment 1 suggest that, with training in the force field, the brain learns a new long-latency feedback controller: this controller responds earlier and more vigorously to perturbations that push the hand in the direction of the field and is 
A

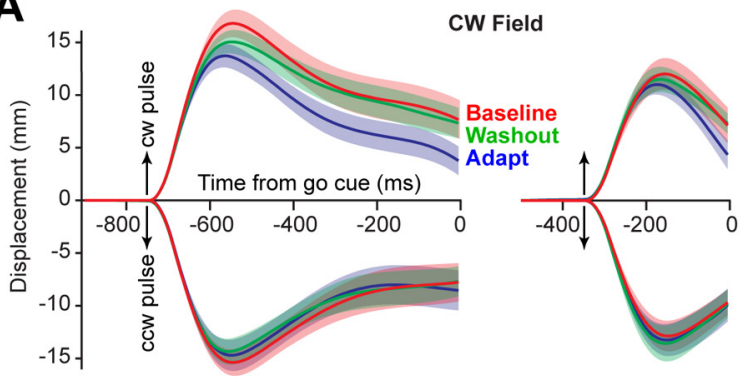

B

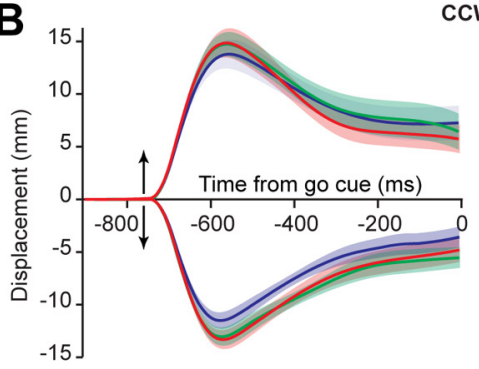

CCW Field

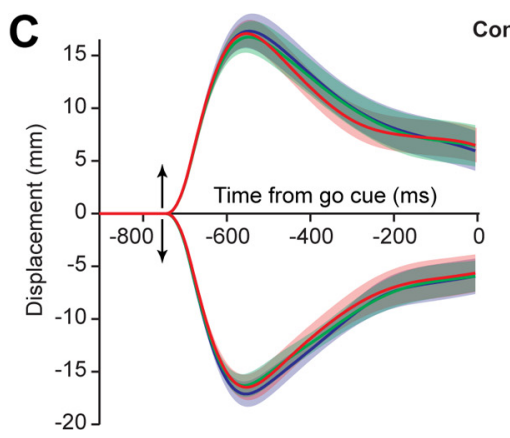

Control
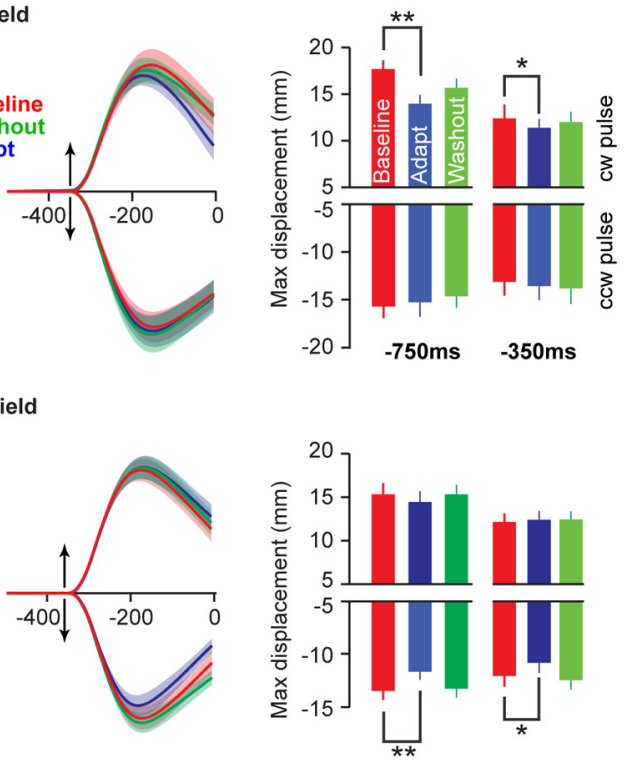

we compared the feedback response during the preparatory period with respect to the baseline condition.

When subjects were preparing to reach to target 1 , an L-pulse produced a smaller displacement of the hand in the adapt versus the baseline conditions (Fig. 5D), whereas an R-pulse produced similar displacements in the two conditions. When subjects were preparing to reach to target 2 , an R-pulse produced a smaller displacement of the hand in the adapt versus the baseline conditions, whereas an L-pulse produced similar displacements in the two conditions. A three-way ANOVA was performed with the following factors: target direction ( 1 or 2 ), condition (baseline or adapt), and pulse (R or L). We found a significant interaction between target direction and pulse $(F=4.99 ; p<0.05)$. Post hoc Tukey's test showed that there was a significant difference between adapt and baseline displacement of the hand to the L-pulse for target $1(p<0.05)$ and R-pulse for target $2(p<0.05)$ (Fig. $5 F)$. Figure $5 E$ shows across-subject average hand position, sampled at $10 \mathrm{~ms}$ intervals. Note that the movement toward the target does not begin until $200 \mathrm{~ms}$ or more after pulse onset. This is important because it demonstrates that changes in EMG and feedback gains that we might see in the period of $0-150 \mathrm{~ms}$ after the pulse are due to a feedback response and not release of motor commands that move the arm toward the target.

Next, we subtracted the EMG responses to the pulses in the adapt condition from the responses in the baseline condition (Fig. 5G). We performed a twoway ANOVA with main factors of target direction and pulse direction and found a

recruited in the preparatory period before the reach. We next performed a second experiment to test whether subjects could learn to contextually select this feedback controller. That is, we wanted to know whether the brain could learn two different feedback controllers and select from among them based on contextual cues. In Experiment 2, our subjects reached to two targets (Fig. 5A). Reaching to target 1 encountered a field that pushed the hand to the left, producing flexion of the elbow and requiring increased activation in the triceps muscle. Reaching to target 2 encountered a field that pushed the hand to the right, producing extension of the elbow and requiring increased activation in the biceps muscle. We wondered whether in the preparatory period before reaching to targets 1 or 2, the brain would engage a feedback controller that would have an increased gain specific to the direction of the upcoming field.

Subjects were presented with a target, and then $450 \mathrm{~ms}$ before the go cue we randomly imposed a pulse in either $45^{\circ}$ (R-pulse, extending the elbow) or $225^{\circ}$ (L-pulse, flexing the elbow). The training schedule is illustrated in Figure $5 B$, and the resulting reach errors are shown in Figure 5C. Reach errors had stabilized to near-baseline levels by the final set of training, at which point significant interaction, suggesting that the pulse direction produced an EMG response that depended on target direction. Post hoc analysis showed that, for target 1, an L-pulse produced increased triceps activity at 45-75 and 75-100 ms delay $(p<0.05)$, whereas an R-pulse produced decreased biceps activity at 75100 ms delay $(p<0.05)$. Post hoc analysis showed that, for target 2, an R-pulse produced increased biceps activity at $20-45$ and $45-75$ ms delay $(p<0.05)$, whereas an L-pulse produced no significant changes at any delay for triceps. Overall, the results of Experiment 2 illustrated that feedback responses were contextually modulated during the preparatory period before reaching to each target.

The EMG analyses that we have reported thus far focused on responses generated to pulses and measured the change in these responses from baseline to adapt conditions. To determine whether the general cocontraction level of muscles had changed with adaptation, we focused on the period before the pulses: 950 $750 \mathrm{~ms}$ before the go cue. For both Experiment 1 and Experiment 2 , we found no muscle that showed a significant increase in activation levels ( $t$ test, all $p>0.05$ ) from baseline to adapt. However, in Experiment 1 for the CCW field we found that biceps tended to 
A

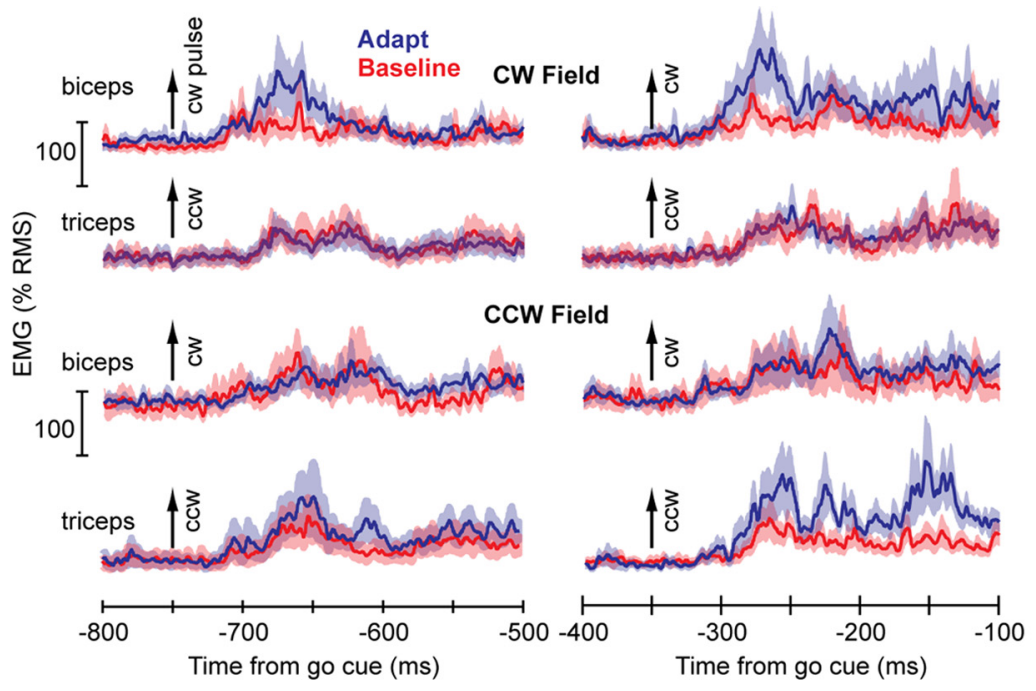

C

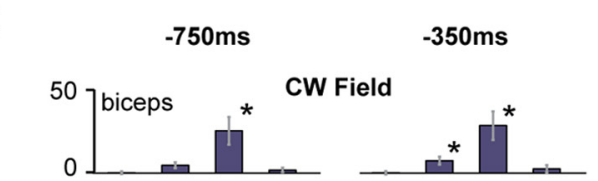

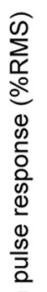

25 triceps
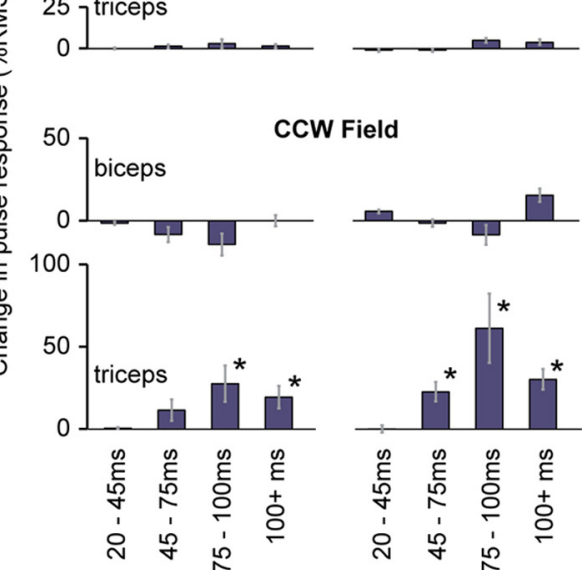

B

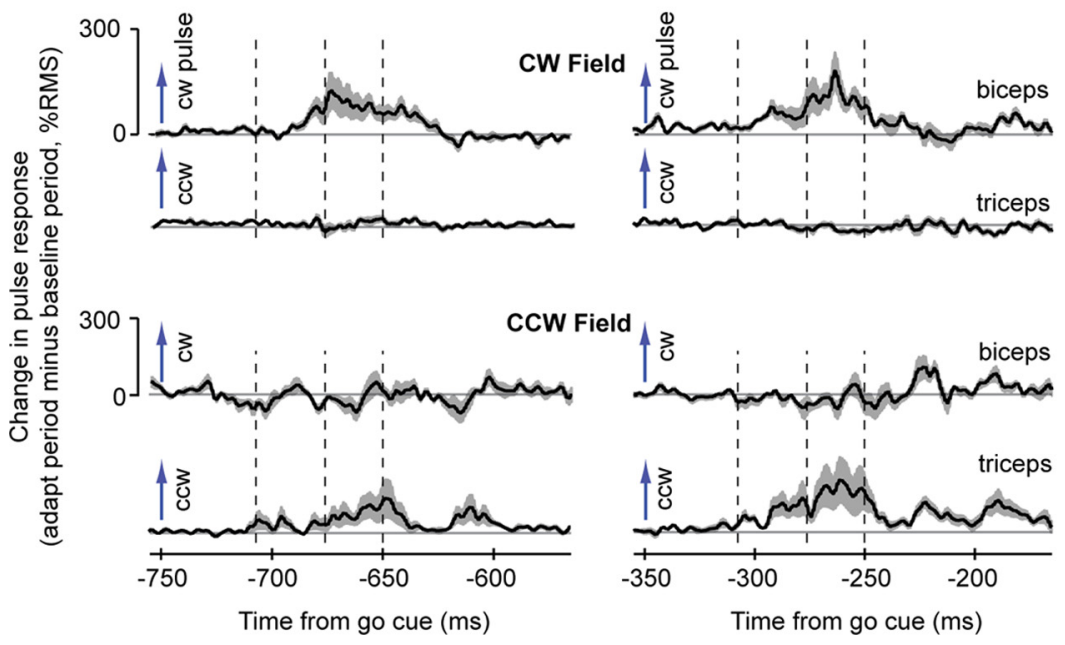

Figure 4. EMG responses to force pulses in the preparatory period. $A$, Across-subject averaged EMG responses in the $C W$ and $C C W$ groups in the baseline and adapt conditions. The response to a CW pulse in biceps and CCW pulse in triceps are plotted. The arrows indicate onset time of the force pulse. The left subfigure is for the $-750 \mathrm{~ms}$ pulse, and the right subfigure is for the $-350 \mathrm{~ms}$ pulse. $\boldsymbol{B}$, Within-subject change in EMG in the adapt condition with respect to baseline. The traces are within-subject changes, comparing pulsed trials in the adapt condition with pulsed trials in the baseline condition. The dashed lines indicate periods $45-75$ and $75-100 \mathrm{~ms}$ after the pulse. C, Same data as in $\boldsymbol{B}$, but binned in time to reflect short-, medium-, and long-latency within-subject EMG changes in reflex responses. Error bars in all plots are SEM. The $p$ values of $t$ tests are indicated by an asterisk: ${ }^{*} p<0.05$.

show a decrease $(t$ test, $p<0.05)$. This result was specific to the preparatory period for subjects who trained in the CCW field and was not present in the CW group. This is interesting because it shows that, in the preparatory period, the muscle that is not involved in responding to the upcoming field is slightly less active in the adapt condition with respect to baseline. In Experiment 2, we also compared the preparatory activity in the adapt condition with respect to baseline. For both target 1 and target 2 , there were slight decreases in biceps and triceps, but these decreases were not target specific. In summary, our comparison of EMG during the preparatory period found no case in which activations increased from baseline to adapt condition. However, in Experiment 1, we did see a field-dependent decrease in the activity of biceps muscle in the preparatory period.

\section{Discussion}

Let us hypothesize that, to generate a movement, the brain computes a feedback controller, transforming sensory states into motor commands. A proxy for this transformation is the gain of the feedback loop, measured in terms of motor commands that are produced in response to a given sensory input. During a reach, this gain is specific to the constraints of the task. For example, when people practice reaching in a curl field that pushes the hand in a CW direction, they increase the gain of their response to a CW perturbation (Wang et al., 2001). If the field is present only in a specific region, the feedback gain during the reach changes immediately before the hand enters that region (Kimura et al., 2006; Kimura and Gomi, 2009). If the field perturbs the hand but this perturbation is irrelevant to success of the task, the feedback gain is decreased during the reach (Scheidt et al., 2005). Here, we wondered whether preparing for a reach involved "loading" of the feedback controller that would be used during the reach. If so, responses to sensory input during the preparatory period should change as the controller adapts.

In Experiment 1, we trained subjects to reach in an environment in which a CW or a CCW curl force field was present. During the preparatory period before the reach, we gave a force pulse to the hand. We found that, if the pulse was in the same direction as the field, the result was a smaller displacement of the 
A

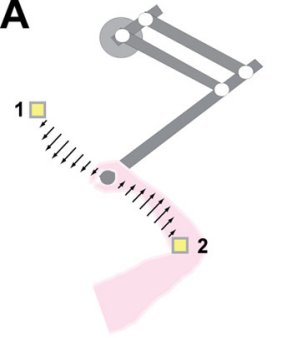

B

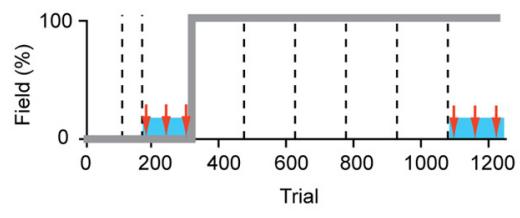

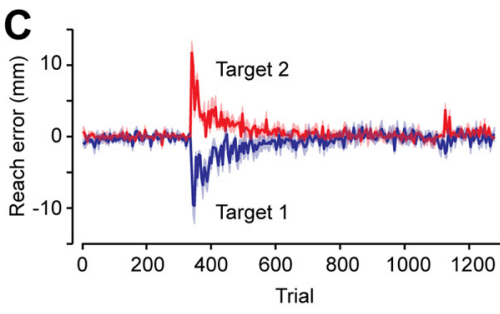

D

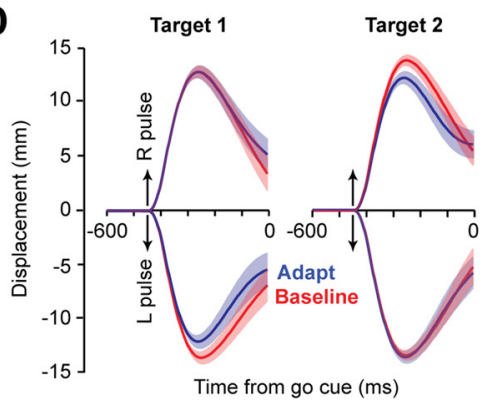

G

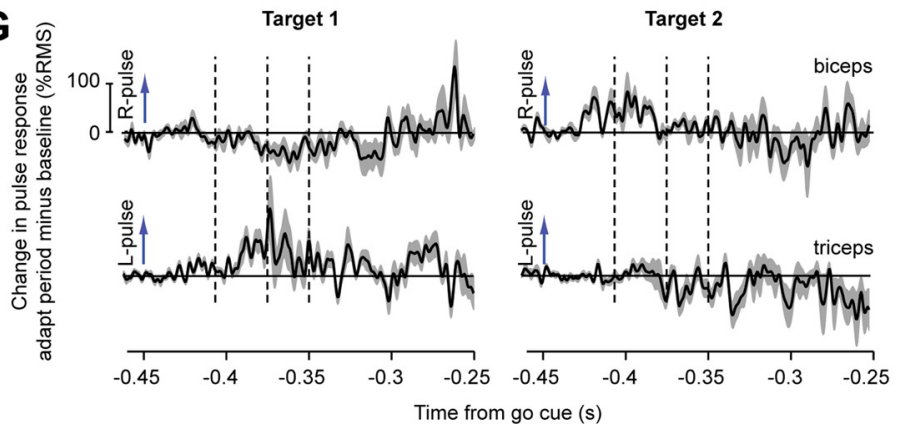

E

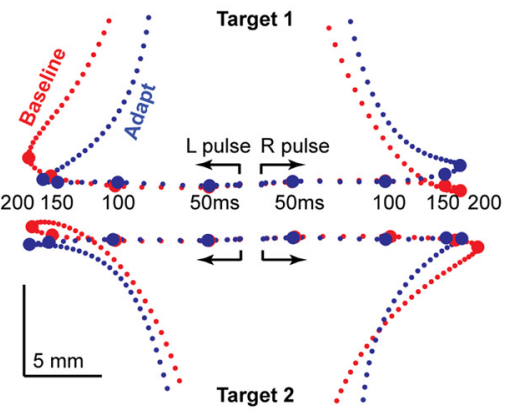

$\mathbf{F}$

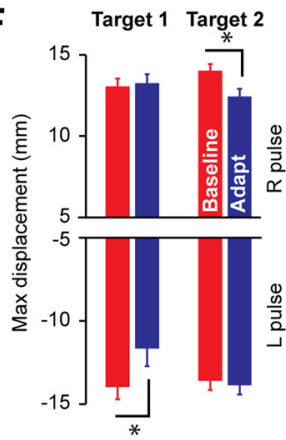

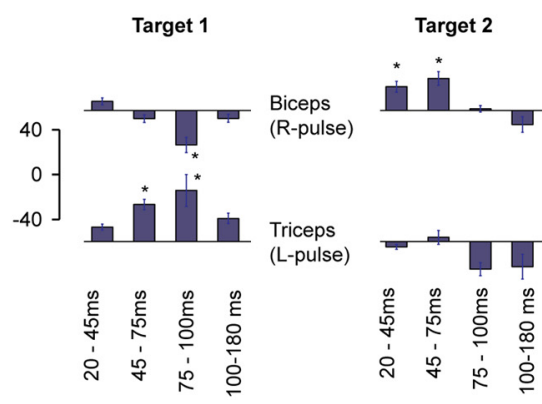

Figure 5. Experiment 2: context-dependent changes in the feedback response. $\boldsymbol{A}$, Experimental setup. Subjects reached to two targets. Reach to target 1 involved interaction with a field that flexed the elbow, whereas reach to target 2 involved interaction with a field that extended the elbow. $\boldsymbol{B}$, Training protocol. We measured feedback response of the arm during the preparatory period in the period marked with red arrows. C, Reach errors (maximum displacement perpendicular to the target direction) for each target. Bin size is 5. The shaded region is SEM. $E$, Hand displacement in response to force pulses in the preparatory period before the reach. L-pulse refers to the force pulse directed toward $225^{\circ}$. R-pulse refers to force pulse directed toward $45^{\circ}$. Displacements were measured parallel to the direction of the force pulse. The shaded region is SEM. $\boldsymbol{D}$, Hand trajectories from pulse trials. The dots represent across-subject average hand position, sampled at $10 \mathrm{~ms}$ intervals. The filled circles identify hand position at $50 \mathrm{~ms}$ intervals. Importantly, note that the movement toward the target does not begin until $200 \mathrm{~ms}$ or more after pulse onset. $\boldsymbol{F}$, Maximum displacement in the baseline and adapt conditions in response to the force pulse. Error bars are SEM. G, Within-subject change in EMG response to force pulses in the adapt condition with respect to baseline. The data were binned in time to reflect short-, medium-, and long-latency within-subject EMG changes. Error bars are SEM. The $p$ values of $t$ tests are indicated by an asterisk: ${ }^{*} p<0.05$.

hand compared with the baseline condition. When the pulse and the field were in the opposite directions, the displacement was unchanged from baseline. In Experiment 2, we trained subjects to reach to two targets and imposed a field that extended the elbow for one target and flexed the elbow for the other target. We found that, after adaptation, as subjects prepared to reach to target 1 they loaded a different feedback gain compared with when they prepared to reach to target 2 . In the preparatory period before reaching to each target, the gain of the feedback loop was higher for perturbations that displaced the hand in the same direction as the field for that target. This contextually selected change in gain was generally present in the long-latency period (45-100 ms latency), but in one instance was also present in the short-latency period (20-45 ms latency). Therefore, with training the brain altered the map that associated sensory feedback with motor commands during the reach, and this map was partially invoked during the preparatory period before the reach.

There are a number of possible pitfalls to our experimental design. (1) During adaptation, there is increased activation of certain muscles during the reach. It is possible that this increased activation alters the sensitivity of the motor neurons that control those muscles, making it so that a given input produces a greater response. This history effect is inconsistent with the results of Experiment 2. (2) It is possible that, with adaptation to a certain field, spindle afferents in the muscles that are involved in compensating for the field become sensitized, producing a greater response for a given stretch. This possibility is also inconsistent with the results of Experiment 2. (3) It is possible that, in anticipation of making the reach, the descending projections to gamma motor neurons are changed to alter the response properties of the spinal stretch reflex circuitry. This would predict increased EMG response in the short-latency period of the adapt condition versus baseline. Although we cannot eliminate this possibility as on one occasion we did find increased responses in the short-latency period, this was a rare observation. (4) Another possibility is that, during adaptation, the increased use of the muscle that counters the field may result in its fatigue. In a fatigued state of a muscle, the shortlatency EMG responses are decreased but the long-latency responses are unchanged (Duchateau and Hainaut, 1993). We observed the opposite effect: an increase in the long-latency response.

A consistent observation in our work and a number of earlier works is that, regardless of whether the perturbation is given 
during the reach or in the preparatory period before the reach, adaptation to a curl field alters the feedback response at $\sim 80 \mathrm{~ms}$ after the perturbation (Wang et al., 2001; Kimura et al., 2006; Kimura and Gomi, 2009). An exception is a report by Wagner and Smith (2008) in which the change in the feedback response was detected $200 \mathrm{~ms}$ after the perturbation. Feedback responses beyond $120 \mathrm{~ms}$ are labeled as voluntary, whereas the shorter latency responses are thought to be involuntary. Whereas in our work here (as well as earlier works cited) the perturbations were parallel to the force field and produced an adapted long-latency response only if the perturbation and the field were in the same direction, in the study by Wagner and Smith (2008) the perturbations were perpendicular to the field.

Previous works have found that long-latency but not shortlatency feedback responses take into account dynamics of the arm (Lacquaniti and Soechting, 1984; Gielen et al., 1988). For example, during posture (Kurtzer et al., 2008) as well as during reaching (Kurtzer et al., 2009) the short-latency response to a shoulder perturbation depends only on the state of the shoulder, whereas the long-latency response depends also on the state of the elbow. Pruszynski et al. (2008) trained people to start their reach via a pulse that pushed the hand toward or away from a target. When the pulse pushed the hand toward the target, the long-latency gain was suppressed, but not the short-latency gain. Therefore, the longlatency responses were modified if the perturbation helped achieve the goal of the task. Similarly, Bonnet (1983) found that, during the preparatory period before a wrist extension, a pulse that extended the wrist produced a depressed long-latency response. What is the neural basis of this adaptable long-latency feedback system? In humans, stimulation of the brain in both healthy (Palmer and Ashby, 1992) and patient populations [an interesting example is from patients with "mirror movements " (Capaday et al., 1991)] suggests that the motor cortex is a critical node in this loop (for a historical review, see Matthews, 1991). In monkeys, the importance of the primary motor cortex in transforming sensory information into rapid motor responses has been noted (Pruszynski et al., 2011). In reach adaptation to force fields, the long-latency feedback of the arm as measured during reaching changes, but this change is eliminated if the motor cortex is stimulated via transcranial magnetic stimulation as the reach begins (Kimura et al., 2006). Therefore, changes in longlatency feedback as measured during reaching likely depend on adaptation that relies on the motor cortex.

What may be the neural basis of the adaptable long-latency feedback during the preparatory period? Evarts and Tanji (1974) recorded from the motor cortex in a task in which a color cue instructed the monkey to either push against or with a force pulse. They found that, within $200 \mathrm{~ms}$ after the instruction, motor cortex activity but not activity in the somatosensory areas of the cortex changed without changes in muscle activity (Tanji and Evarts, 1976). Importantly, when the pulse was given, the prior instruction produced a $20-25 \mathrm{~ms}$ response in the motor cortex that was instruction invariant, and then a $40-50 \mathrm{~ms}$ response that depended on the prior instruction (Evarts and Tanji, 1976). Therefore, contextual information modulates activity of motor cortex in the preparatory period before a voluntary movement, and that information affects response of motor cortical cells to arm perturbations at a latency of $\sim 45 \mathrm{~ms}$ but not earlier. In this framework, the observation that adaptation altered the longlatency response appears consistent with the hypothesis that cortical control of the reach was altered, and this change was reflected in the feedback response during the preparatory period.

During force field adaptation, activities of neurons in the supplementary motor area (SMA) and dorsal premotor cortex
(PMd) change in the preparatory period (with respect to a preadaptation condition), but not in ventral premotor cortex (PMv) or primary motor cortex (M1) (Padoa-Schioppa et al., 2002). One possibility is that, during the preparatory period, SMA and PMd transform a kinematic plan into a plan that takes into account dynamics of the task, and then that plan is executed by M1 after the go cue is provided (Padoa-Schioppa et al., 2002). However, Churchland et al. (2010) noted that the delay period activity is not a smaller or subthreshold version of the activity of the cell during the movement. In light of our results, it seems possible that the activities in SMA and PMd during the delay period are not related to the motor commands that are planned for the upcoming movement, but at least partly responsible for setting up the feedback gains that will be present during the movement. Our work here raises the possibility that one function of preparatory motor cortical activity is to place the cortical network in a state that implements the feedback gains appropriate for the upcoming reaching movement.

\section{References}

Bonnet M (1983) Anticipatory changes of long-latency stretch responses during preparation for directional hand movements. Brain Res 280:51-62.

Burdet E, Osu R, Franklin DW, Milner TE, Kawato M (2001) The central nervous system stabilizes unstable dynamics by learning optimal impedance. Nature 414:446-449.

Capaday C, Forget R, Fraser R, Lamarre Y (1991) Evidence for a contribution of the motor cortex to the long-latency stretch reflex of the human thumb. J Physiol 440:243-255.

Carlsen AN, Mackinnon CD (2010) Motor preparation is modulated by the resolution of the response timing information. Brain Res 1322:38-49.

Carlsen AN, Chua R, Timothy Inglis J, Sanderson DJ, Franks IM (2008) Motor preparation in an anticipation-timing task. Exp Brain Res 190:453-461.

Churchland MM, Cunningham JP, Kaufman MT, Ryu SI, Shenoy KV (2010) Cortical preparatory activity: representation of movement or first cog in a dynamical machine? Neuron 68:387-400.

Diedrichsen J (2007) Optimal task-dependent changes of bimanual feedback control and adaptation. Curr Biol 17:1675-1679.

Duchateau J, Hainaut K (1993) Behaviour of short and long latency reflexes in fatigued human muscles. J Physiol 471:787-799.

Evarts EV, Tanji J (1974) Gating of motor cortex reflexes by prior instruction. Brain Res 71:479-494.

Evarts EV, Tanji J (1976) Reflex and intended responses in motor cortex pyramidal tract neurons of monkey. J Neurophysiol 39:1069-1080.

Franklin DW, Liaw G, Milner TE, Osu R, Burdet E, Kawato M (2007) Endpoint stiffness of the arm is directionally tuned to instability in the environment. J Neurosci 27:7705-7716.

Gielen CC, Ramaekers L, van Zuylen EJ (1988) Long-latency stretch reflexes as co-ordinated functional responses in man. J Physiol 407:275-292.

Kimura T, Gomi H (2009) Temporal development of anticipatory reflex modulation to dynamical interactions during arm movement. J Neurophysiol 102:2220-2231.

Kimura T, Haggard P, Gomi H (2006) Transcranial magnetic stimulation over sensorimotor cortex disrupts anticipatory reflex gain modulation for skilled action. J Neurosci 26:9272-9281.

Kurtzer IL, Pruszynski JA, Scott SH (2008) Long-latency reflexes of the human arm reflect an internal model of limb dynamics. Curr Biol 18:449-453.

Kurtzer I, Pruszynski JA, Scott SH (2009) Long-latency responses during reaching account for the mechanical interaction between the shoulder and elbow joints. J Neurophysiol 102:3004-3015.

Lacquaniti F, Soechting JF (1984) Behavior of the stretch reflex in a multijointed limb. Brain Res 311:161-166.

Matthews PB (1991) The human stretch reflex and the motor cortex. Trends Neurosci 14:87-91.

Padoa-Schioppa C, Li CS, Bizzi E (2002) Neuronal correlates of kinematicsto-dynamics transformation in the supplementary motor area. Neuron 36:751-765.

Palmer E, Ashby P (1992) Evidence that a long latency stretch reflex in humans is transcortical. J Physiol 449:429-440. 
Pruszynski JA, Kurtzer I, Scott SH (2008) Rapid motor responses are appropriately tuned to the metrics of a visuospatial task. J Neurophysiol 100:224-238.

Pruszynski JA, Kurtzer I, Nashed JY, Omrani M, Brouwer B, Scott SH (2011) Primary motor cortex underlies multi-joint integration for fast feedback control. Nature 478:387-390.

Scheidt RA, Conditt MA, Secco EL, Mussa-Ivaldi FA (2005) Interaction of visual and proprioceptive feedback during adaptation of human reaching movements. J Neurophysiol 93:3200-3213.

Shadmehr R, Krakauer JW (2008) A computational neuroanatomy for motor control. Exp Brain Res 185:359-381.

Tanji J, Evarts EV (1976) Anticipatory activity of motor cortex neurons in relation to direction of an intended movement. J Neurophysiol 39:1062-1068.

Todorov E, Jordan MI (2002) Optimal feedback control as a theory of motor coordination. Nat Neurosci 5:1226-1235.

Wagner MJ, Smith MA (2008) Shared internal models for feedforward and feedback control. J Neurosci 28:10663-10673.

Wang T, Dordevic GS, Shadmehr R (2001) Learning the dynamics of reaching movements results in the modification of arm impedance and longlatency perturbation responses. Biol Cybern 85:437-448.

Wong J, Wilson ET, Malfait N, Gribble PL (2009) The influence of visual perturbations on the neural control of limb stiffness. J Neurophysiol 101: $246-257$. 